\title{
Copy Choice Mechanism of Immunoglobulin Heavy-Chain Switch Recombination
}

\author{
WESLEY DUNNICK ${ }^{1 *}$ AND JANET STAVNEZER ${ }^{2}$ \\ Department of Microbiology and Immunology, University of Michigan Medical School, Ann Arbor, \\ Michigan 48109-0620, ${ }^{1}$ and Department of Molecular Genetics and Microbiology, University of Massachusetts \\ Medical School, Worcester, Massachusetts $01655^{2}$
}

Received 3 July 1989/Accepted 9 October 1989

\begin{abstract}
The immunoglobulin heavy-chain switch is mediated by a recombination event between DNA switch regions associated with donor and recipient constant-region genes. We have determined that the mutations which can be found in some switch regions after recombination appear to arise on only one strand of DNA. This result suggests that switch recombination involves error-prone synthesis of one DNA strand and ligation of the other strand from preexisting DNA.
\end{abstract}

The immunoglobulin heavy-chain switch is mediated by a DNA recombination event between switch regions $(2-4,8$, 16). Switch regions are located a few kilobases upstream of each heavy-chain constant-region coding segment and are composed of simple sequences repeated in tandem $(5,6,10$, 18). Recombination between a donor (for example, $S \mu$ ) and a recipient (for example, $S \alpha$ ) switch region results in deletion of the intervening DNA, with its constant-region coding segments, and in physical linkage of the variable and recipient constant-region coding segments. A functional connection between DNA synthesis and heavy-chain switching has been suggested by several studies $(11,20,25)$. Other forms of nonhomologous recombination probably take place by a copy choice mechanism (1). We suggested that DNA synthesis is directly involved in switch recombination because it accounts for deletions, duplications, and mutations found in recombined switch regions (7). To understand the mechanism of DNA synthesis during switch recombination, we have now determined the sequence of an $S \mu$ region before switch recombination to $S \alpha$. Comparison of this sequence with that of recombined $S \mu S \alpha$ genes from clonally related cells reveals that some progeny of a single-switch recombination event have mutations, whereas other progeny do not. This result is consistent with a model in which one strand of DNA is synthesized during the switch recombination event.

Cell lines and molecular clones. Immunoglobulin M-positive $(\mathrm{IgM}+) \mathrm{I} .29$ cells were prepared by fluorescence-activated cell sorting and were passaged through mice five times as described previously (22). Each population of cells was named according to the number of passages: for example, the I.29 IgM.1P $(99 \% \mu+, 37 \% \alpha+)$ cells had been passaged through mice once and IgM.5P cells $(17 \% \mu+, 77 \% \alpha+)$ had been passaged through mice five times. I. $29 \mu$ cells were also adapted to tissue culture, in which switching to $\alpha$ expression could be induced by treatment with anti-idiotype antibodies and lipopolysaccharide or by anti-IgM and lipopolysaccharide (23).

Various molecular clones were derived from these I.29 cell populations (Fig. 1). The molecular clone $\mathrm{E} \mu .5$ represents the expressed $\mu$ heavy-chain gene from the I.29 cell line IgM.1P (12). The $S \mu$ region is prone to deletion in bacteriophage vectors; the $E \mu .5$ phage population included phage with $S \mu$ regions ranging in size from 0.7 to 2.5

\footnotetext{
* Corresponding author.
}

kilobases, the largest $S \mu$ region being the same size as the genomic $S \mu$. From the IgM.5P DNA, we derived molecular clones containing expressed $\alpha$ genes with $S \mu S \alpha$ recombina-

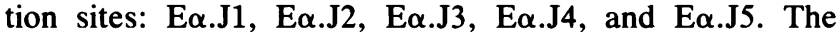
molecular clones BFO.5 and BFO.6 represent independent isolates of the expressed $\alpha$ gene from I.29 cells that switched in vivo and were cloned in vitro (21). 41.3 is a molecular clone of the expressed $\alpha$ gene from I.29 $\mu$ cells that had been induced to switch in vitro. These $\mathrm{S} \mu \mathrm{S} \alpha$-containing clones have been described $(7,12,23)$.

DNA sequence of an $S \mu$ region from a $\mu$-expressing $I .29$ cell line. In the process of subcloning the $S \mu$ region from $E \mu .5$ into the Bluescript $\mathrm{KS}^{-}$vector (Stratagene, La Jolla, Calif.), we obtained a plasmid clone with a $2.5-\mathrm{kb}$ SstI-HindIII fragment, which includes an apparently intact $S_{\mu}$ region. Fragments from Sst I-HindIII, BstEII, and Bst NI digests and from partial $A l u I$ digests were further subcloned into M13mp18 and M13mp19 and sequenced by the dideoxy method (19). The sequence of the $S \mu$ region in the molecular clone $\mathrm{E}_{\mu} .5$ was determined and found to be composed entirely of the pentamers GGGGT and GAGCT or closely related pentamers (e.g., GGGCT and GAGGT). $\mathrm{S} \mu$ sequences in the recombined $S \mu S \alpha$ regions of the clones 41.3, BFO.5, BFO.6, E $\alpha . J 1, E \alpha . J 2, E \alpha . J 3, E \alpha . J 4$, and E $\alpha . J 5$ could be aligned with the $E_{\mu} .5 \mathrm{~S} \mu$ sequence.

Schematic representations of the $S \mu$ sequences of the $\mathrm{E} \mu .5, \mathrm{BFO} .5 / \mathrm{BFO} .6,41.3$, and $\mathrm{E} \alpha . \mathrm{J}$ clones are shown in Fig. 2A. A small portion of each of these sequences, which include $\mathrm{S} \mu \mathrm{S} \alpha$ recombination sites for the BFO.5/BFO.6 and E $\alpha . J$ clones (indicated in Fig. 2A), is presented in Fig. 2B. The E $\alpha . J 1 / E \alpha . J 3 / E \alpha . J 5$, E $\alpha . J 2 / E \alpha . J 4, B F O .5 / B F 0.6$, and $\mathrm{E} \mu .5$ sequences are compared with a consensus $\mathrm{S} \mu$ se-

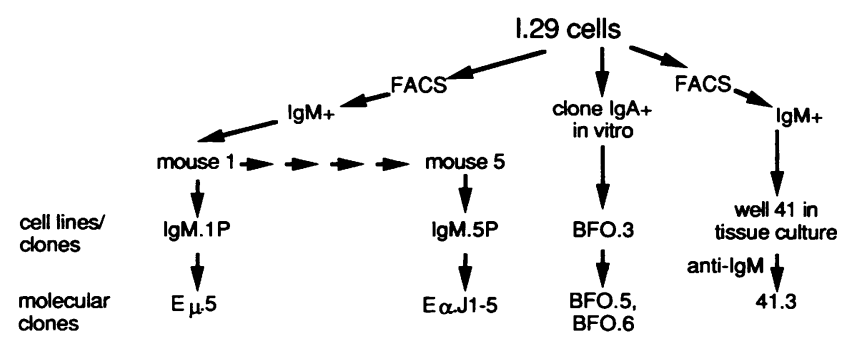

FIG. 1. Derivation of I.29 cell and molecular clones. See text for explanation. 

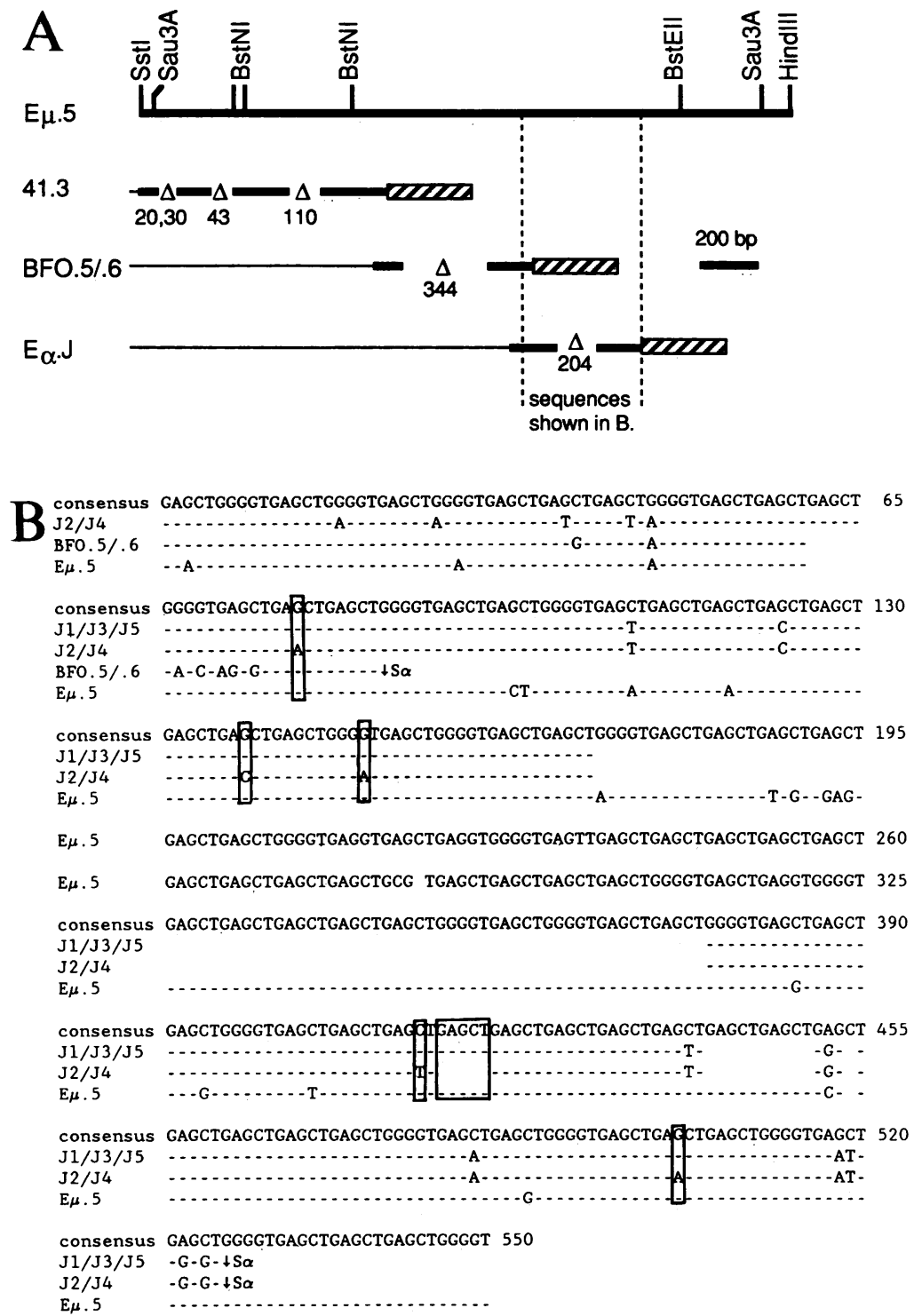

FIG. 2. $S \mu$ sequences in $S \mu$ and $S \mu S \alpha$ genes from I.29 cell lines. (A) Schematic diagrams of the $S \mu$ sequences in various I.29 clones are shown. For $\mathrm{E} \mu .5$, restriction enzyme cleavage sites used in determining the complete DNA sequence of the $S \mu$ region of this molecular clone are shown. The 2,541-bp sequence of the $S \mu$ region of $E \mu .5$ has been deposited with GenBank under accession number M28469. S $\mu$ sequences of the recombinant $S_{\mu} S \alpha$ regions in 41.3, BFO.5/BFO.6, and the E $\alpha . J$ series of clones (7) are schematically diagrammed to show the relationships between these $S \mu$ regions and that in $E \mu$.5. The thick solid lines indicate the portions of $S \mu$ that have been sequenced; the thin horizontal lines indicate portions of $S \mu$ that have not been sequenced. The hatched boxes indicate $S \alpha$ sequences. The spaces with deltas indicate deletions in the $\mathrm{S} \mu \mathrm{S} \alpha$ regions relative to $\mathrm{E} \mu .5$, with the length of the deletion in base pairs indicated beneath the delta. The $5^{\prime}$-most deletion site indicated in 41.3 (with 20-and 30-bp deletions) is complex and also includes additional 5- and 15-bp deletions. The portion of S $\mu$ sequences presented in part B is indicated by dashed vertical lines. (B) Nucleotide sequences of $S \mu$ regions. S $\mu$ sequences from the indicated molecular clones are compared with a consensus $S \mu$ sequence composed of only GGGGT and GAGCT pentamers. Differences from the consensus are indicated by the appropriate base abbreviation; identity is indicated by a dash. Deletions relative to the consensus sequence are indicated by spaces. To conserve space, part of the $\mathrm{E} \mu .5$ sequence is not compared with a consensus sequence and is presented as a single line (residues 196 through 325). Differences between the E $\alpha . J 1 / 3 / 5$ and the E $\alpha . J 2 / 4$ sequences are noted by boxes.

quence composed only of the pentamers GGGGT and GAGCT. Relative to the E $\mu .5$ sequence, the E $\alpha$.J $S \mu$ regions have apparently suffered a 204-base-pair (bp) deletion (residues 171 through 375). In the region of $S \mu$ sequence not shown in Fig. 2B, the BFO.5/BFO.6 sequence has a 344-bp deletion relative to the $\mathrm{E} \mu .5 \mathrm{~S} \mu$ sequence. The 41.3 sequence has six different deletions of $110,43,30,20,15$, and 5 bp relative to the $\mathrm{E} \mu .5$ (Fig. $2 \mathrm{~A}$ ). The deletions in the E $\alpha . \mathrm{J}$ and BFO.5/BFO.6 clones were observed in independent bacteriophage clones that represented the same $S \mu S \alpha$ region; thus, they are unlikely to be the result of cloning artifacts.

The E $\alpha . J$ series of molecular clones was derived from one cell population, and these clones share the same $S \mu S \alpha$ recombination site; they are apparently the result of a single-switch recombination event. Nevertheless, the sequences of the $S \mu S \alpha$ regions of these clones fall into two groups $(\mathrm{E} \alpha . \mathrm{J} 1 / \mathrm{E} \alpha . \mathrm{J} 3 / \mathrm{E} \alpha . \mathrm{J} 5$ and $\mathrm{E} \alpha . \mathrm{J} 2 / \mathrm{E} \alpha . \mathrm{J} 4)$ on the basis of 
A.

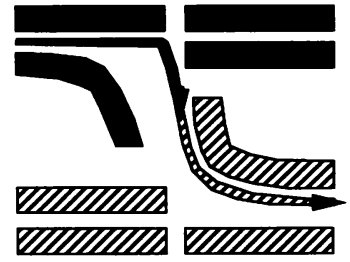

B.
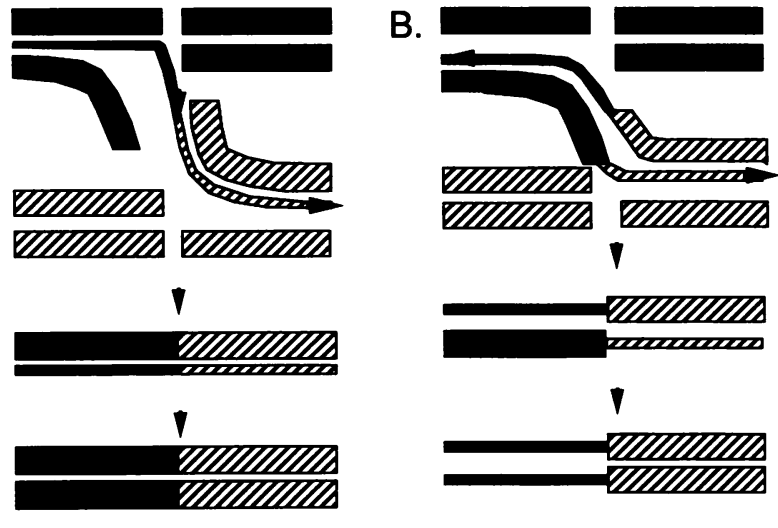

and

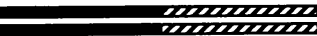

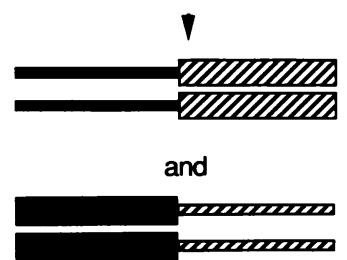

FIG. 3. DNA synthesis in heavy-chain switch recombination. Two mechanisms that are consistent with our results are shown. In mechanism A, DNA synthesis, indicated by thin curves with arrows, of one of the strands of the $S \mu$ region (solid boxes) switches templates at a DNA breakpoint in the $S \mu$ region to a breakpoint in the $S \alpha$ region (hatched boxes). Switch recombination is partially completed by copy choice DNA replication and is completed by a ligation reaction of old (thick boxes) strands. After recombination is complete, the hybrid switch region is a heteroduplex, with old strands (thick boxes) from both the $S_{\mu}$ and $S \alpha$ regions and new strands (thin boxes) from both regions. In the next cell division, this heteroduplex will be duplicated, so that in some switch regions, germ line sequences will be observed (from the old strands) and in other switch regions, mutations will be observed (from the new strands). In mechanism $B$, DNA synthesis of the $S \mu$ region is primed by an $S \alpha$ DNA strand and DNA synthesis of the $S \alpha$ region is primed by an $S \mu$ strand. Recombination is completed by a ligation reaction. After duplication of the resulting heteroduplex, two products will be observed, both having new strands in one part of the recombinant switch region and old strands in the other part of the recombinant switch region. As discussed in the text, these models are consistent with the pattern of mutations in the $S \mu$ region of the E $\alpha . J$ series of clones; the pattern of mutations and duplication in the $S \alpha$ region is too complex to be informative.

single base changes and small deletions (7). Examples of these differences are shown in Fig. 2B. In 240 bp of $S \mu$ sequence, $\mathrm{E} \alpha . \mathrm{J} 1 / \mathrm{E} \alpha . \mathrm{J} 3 / \mathrm{E} \alpha . \mathrm{J} 5$ differ from $\mathrm{E} \alpha . \mathrm{J} 2 / \mathrm{E} \alpha . \mathrm{J} 4$ at five positions and by a 5-bp deletion (noted by boxes). At these differences, the $\mathrm{E} \alpha . \mathrm{J} 1 / \mathrm{E} \alpha . \mathrm{J} 3 / \mathrm{E} \alpha . \mathrm{J} 5$ sequences always match the $S \mu$ sequence from the $\mu$-expressing cell $(E \mu .5)$.

Error-prone DNA synthesis of a single strand during switch recombination. The E $\alpha . J$ series of molecular clones represents two groups of progeny of a single-switch recombination event (7). Two models for switch recombination which account for two distinguishable progeny from one recombination event are shown in Fig. 3. In model A, DNA synthesis switches templates between the $S \mu$ and the $S \alpha$ regions. Model A is essentially the same as a previous model for switch recombination (7). In model $B$, a preexisting $S \mu$ strand primes DNA synthesis by using the $S \alpha$ region as a template and vice-versa. We had not considered such a mechanism for switch recombination previously, but it is consistent with existing data. Both models are consistent with DNA sequences of switch recombination sites; template switching (model A) or illegitimate priming (model B) could take place at the short sequences most often found at switch recombination sites (13-15). In addition, template switching or illegitimate priming could take place at different sequences within the same switch region, resulting in the deletions associated with switching we and others observe (Fig. 2) $(7,9,17,23,24)$. Duplication of switch region sequences could arise by DNA synthesis initiating at multiple sites simultaneously (7), rather than at a single site as shown in Fig. 3.

Both of the models presented in Fig. 3 predict that a heteroduplex, $\mu-\alpha$ hybrid switch region is an intermediate of switch recombination. The heteroduplex arises as the old DNA strand pairs with the newly synthesized strand, which includes base replacement errors. In the next cell division, each strand of the heteroduplex would be copied faithfully, resulting in two progeny-one with mutations and one without. Both of the models predict that for the $S \mu$ part of a hybrid $S \mu S \alpha$ region, all of the mutations will be in one set of progeny. At the $S \mu$ residues that are analyzable in the E $\alpha . J$ series of clones, this is the result we observe (Fig. 2B). The region of error-prone DNA synthesis can include at least 300 bp on either side of the switch recombination site; mutations in switch regions can be identified at least this far from the recombination site (7).

The pattern of base substitutions in the E $\alpha . J$ S $\mu$ region leads us to hypothesize DNA synthesis of only one (new) strand in $S \mu$. The $S \alpha$ parts of the E $\alpha . J$ hybrid $S \mu S \alpha$ regions present a different pattern of base substitutions. In the S $\alpha$ region near the $S \mu S \alpha$ recombination site, we have reported that the $\mathrm{E} \alpha . \mathrm{J} 1 / \mathrm{E} \alpha . \mathrm{J} 3 / \mathrm{E} \alpha . \mathrm{J} 5$ sequences differ by four single base changes and a five-nucleotide deletion from the E $\alpha . J 2 /$ E $\alpha . J 4$ sequences (7). The $E \alpha . J 1 / E \alpha . J 3 / E \alpha . J 5$ sequence matched the corresponding $\mathrm{S} \alpha$ germ line sequence at only three of the five mutations; the E $\alpha . J 2 / E \alpha . J 4$ matched the germ line at the other two mutations. However, the $S \mu$ and $S \alpha$ parts of the hybrid switch regions differ in a second way. The E $\alpha . J$ S $\mu$ regions (Fig. 2B) include no obvious tandem duplications relative to the $\mathrm{E} \mu .5$ sequence. The $\mathrm{S} \alpha$ sequences near the $S \mu S \alpha$ recombination site appear to have been generated by tandem duplication of germ line $\mathrm{S} \alpha$ elements (7). In order to duplicate $295 \mathrm{bp}$ of $\mathrm{S} \alpha$ region, both DNA strands must be synthesized, although each of the two 295-bp regions could have one old and one new strand. In addition, there are two $S \alpha$ residues at which all of the E $\alpha . J$ clones are the same but different from the germ line sequence to which they were compared (7). This emphasizes the fact that it is not clear which part of the S $\alpha$ germ line sequence was duplicated in the generation of the E $\alpha . J \mathrm{~S} \mu \mathrm{S} \alpha$ recombinant switch region. The complex nature of the E $\alpha . J$ $\mathrm{S} \alpha$ sequences makes them uninformative with regard to the pattern of DNA synthesis in the heavy-chain switch.

The two sets of E $\alpha . J$ clones often have the same nucleotide at certain residues but differ from the $E \mu .5$ sequence. We interpret these differences as mutations in the E $\mu .5$ switch region, which occurred subsequent to the switch event that produced the IgA+ cell from which the E $\alpha . J$ clones were derived. Perhaps the clone of $\mu$-expressing cells in the IgM.1P population, from which the molecular clone $\mathrm{E} \mu .5$ was derived, attempted to switch from $\mu$ to $\alpha$. During those attempted switches, mutations may have been introduced into the $S \mu$ region. At these mutations, the $\mathrm{E} \mu .5$ sequence would differ from that derived from the IgM.5P cell line, which went through independent switch events and gave rise to the E $\alpha . J$ series of clones.

It is interesting that replication of switch region sequences subsequent to that occurring during switch recombination appears to be quite faithful. Independent IgA + cells have identical $S \mu S \alpha$ sequences (e.g., the cells that gave rise to $E \alpha . J 1, E \alpha . J 3$, and E $\alpha . J 5$ [7]). Thus, the sequences of switch 
regions are not inherently susceptible to mutation, but, rather, the mechanism of switch recombination or the enzyme that effects the switch or both appear to be error prone.

We thank J. Latham Claflin and David Friedman for helpful discussions and Deborah Klein for deriving the $\mathrm{E} \mu .5$ molecular clone.

This work was supported by Public Health Service grants CA39068 (awarded by the National Cancer Institute to W.D.) and AI-23283 (awarded by the National Institute of Allergy and Infectious Disease to J.S.).

\section{LITERATURE CITED}

1. Brunier, D., B. Michel, and S. D. Ehrlich. 1988. Copy choice illegitimate DNA recombination. Cell 52:883-892.

2. Coleclough, C., D. Cooper, and R. P. Perry. 1980. Rearrangement of immunoglobulin heavy chain genes during B-lymphocyte development as revealed by studies of mouse plasmacytoma cells. Proc. Natl. Acad. Sci. USA 77:1422-1426.

3. Cory, S., J. Jackson, and J. M. Adams. 1980. Deletions in the constant region locus can account for switches in immunoglobulin heavy chain expression. Nature (London) 285:450-456.

4. Davis, M. M., K. Calame, P. W. Early, D. L. Livant, R. Joho, I. L. Weissman, and L. Hood. 1980. An immunoglobulin heavy chain gene is formed by at least two recombinational events. Nature (London) 283:733-739.

5. Davis, M. M., S. K. Kim, and L. E. Hood. 1980. DNA sequences mediating class switches in alpha-immunoglobulins. Science 209:1360-1365.

6. Dunnick, W., T. H. Rabbitts, and C. Milstein. 1980. An immunoglobulin deletion mutant with implications for the heavychain switch and RNA splicing. Nature (London) 286:669-675.

7. Dunnick, W., M. Wilson, and J. Stavnezer. 1989. Mutations, duplication, and deletion of recombined switch regions sugges a role for DNA replication in the immunoglobulin heavy chain switch. Mol. Cell. Biol. 9:1850-1856.

8. Honjo, T., and T. Kataoka. 1978. Organization of immunoglobulin heavy chain genes and allelic deletion model. Proc. Natl. Acad. Sci. USA 75:2140-2144.

9. Hurwitz, J. L., and J. J. Cebra. 1982. Rearrangements between the immunoglobulin heavy chain gene $\mathrm{JH}$ and $\mathrm{C} \mu$ regions accompany normal B lymphocyte differentiation in vitro. $\mathrm{Na}$ ture (London) 299:742-744.

10. Kataoka, T., T. Miyata, and T. Honjo. 1981. Repetitive sequences in class-switch recombination regions of immunoglobulin heavy chain genes. Cell 23:357-368.

11. Kenter, A. L., and J. V. Watson. 1987. Cell cycle kinetics model of LPS-stimulated spleen cells correlates switch region rear- rangements with S phase. J. Immunol. Methods 97:111-117.

12. Klein, D., J. Nietupski, S. Sirlin, and J. Stavnezer. 1988. I.29 lymphoma cells express a non-mutated immunoglobulin heavy chain variable region gene before and after heavy chain switch. J. Immunol. 140:1676-1684.

13. Marcu, K. B., R. B. Lang, L. W. Stanton, and L. J. Harris. 1982. A model for the molecular requirements of immunoglobulin heavy chain class switching. Nature (London) 298:87-89.

14. Nikaido, T., Y. Yamawaki-Kataoka, and T. Honjo. 1982. Nucleotide sequences of switch regions of immunoglobulin $\mathrm{C} \varepsilon$ and $\mathrm{C}_{\gamma}$ genes and their comparison. J. Biol. Chem. 257:7322-7329.

15. Petrini, J., and W. Dunnick. 1989. Products and implied mechanism of $\mathrm{H}$ chain switch recombination. J. Immunol. 142: 2932-2935.

16. Rabbitts, T. H., A. Forster, W. Dunnick, and D. L. Bentley. 1980. The role of gene deletion in the immunoglobulin heavy chain switch. Nature (London) 283:351-356.

17. Radbruch, A., and F. Sablitzky. 1983. Deletion of $\mathrm{C} \mu$ genes in mouse B lymphocytes upon stimulation with LPS. EMBO J. 2:1929-1935.

18. Sakano, H., R. Maki, Y. Kurosawa, W. Roeder, and S. Tonegawa. 1980. Two types of somatic recombination are necessary for the generation of complete immunoglobulin heavy chain genes. Nature (London) 286:676-683.

19. Sanger, F., A. R. Coulson, B. G. Barrell, A. J. H. Smith, and B. A. Roe. 1980. Cloning in single stranded bacteriophage as an aid to rapid DNA sequencing. J. Mol. Biol. 143:161-178.

20. Severinson, E., S. Bergstedt-Lindquist, W. Van der Roo, and C. Fernandez. 1982. Characterization of the IgG response induced by polyclonal B cell activators. Immunol. Rev. 67:73-85.

21. Sitia, R., A. Rubartelli, and U. Hammerling. 1981. Expression of two immunoglobulin isotypes, IgM and IgA, with identical idiotype in the B cell lymphoma I.29. J. Immunol. 127:13881394.

22. Stavnezer, J., K. B. Marcu, S. Sirlin, B. Alhadeff, and U. Hammerling. 1982. Rearrangements and deletions of immunoglobulin heavy chain genes in the double-producing B cell lymphoma I.29. Mol. Cell. Biol. 2:1002-1013.

23. Stavnezer, J., S. Sirlin, and J. Abbott. 1985. Induction of immunoglobulin isotype switching in cultured 1.29 lymphoma cells. J. Exp. Med. 161:577-601.

24. Szurek, P., J. Petrini, and W. Dunnick. 1985. Complete nucleotide sequence of the murine $\gamma 3$ switch region and analysis of switch recombination sites in two $\gamma 3$-expressing hybridomas. $\mathrm{J}$. Immunol. 135:620-626.

25. van der Loo, W., E. Severinson-Gronowitz, S. Strober, and L. A. Hertzenberg. 1979. Cell differentiation in the presence of cytochalasin B: studies on the "switch" to IgG secretion after polyclonal B cell activation. J. Immunol. 122:1203-1208. 